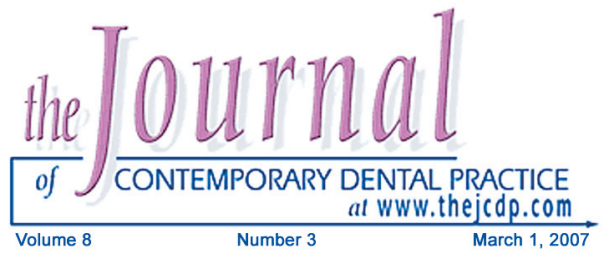

\title{
Prevalence and Severity of Hypertension in a Dental Hygiene Clinic
}

Ana Luz Thompson, RDH, MHE; Marie A. Collins, RDH, EdD;

Mary Cannon Downey, RDH, MS; Wayne W. Herman, DDS, MS;

Joseph Louis Konzelman Jr., DDS; Sue Tucker Ward, RDH, Med;

Cynthia T. Hughes, RDH, BSDH, MEd

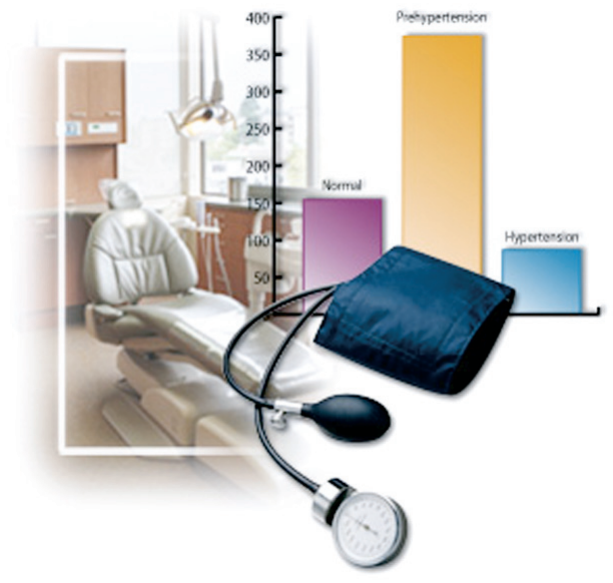

\section{Abstract}

Aim: The purpose of this study was to assess the prevalence and severity of hypertension in a dental hygiene clinic and evaluate factors related to the disease.

Methods and Materials: Records of 615 patients, treated by dental hygiene students during 2003, were reviewed. Data collected included systolic and diastolic blood pressure, presence of diabetes and renal disease, non-modifiers (race, gender, and age), and modifiers (marital status, smoking habits, and occupation).

Results: According to the Seventh Report of the Joint National Committee on the Prevention, Detection, Evaluation, and Treatment of High Blood Pressure (JNC7) classification, $154(25 \%)$ of the subjects had normal blood pressure readings, $374(60.8 \%)$ had prehypertension, and $87(14.1 \%)$ had stage 1 hypertension. Statistical analysis showed a significant difference in the $\mathrm{JNC7}$ classification between groups when considering the non-modifiers' race $(p=.02)$ and the modifiers' smoking habits $(p=.03)$ and occupation $(p=.01)$. A statistically significant difference in the JNC7 classification existed between groups with diabetes $(p=.00)$. The majority of patients had blood pressure readings in the prehypertension stage.

Conclusion: Based on these results, the researchers recommend clinical policy modifications which include: additional documentation for blood pressure readings in the prehypertension stage, lowering the systolic

(C) Seer Publishing 
reading from $160 \mathrm{mmHg}$ to $140 \mathrm{mmHg}$ when adding hypertension alert labels, and noting prehypertension/ hypertension on the dental hygiene care plan with the appropriate interventions.

Keywords: Hypertension, JNC7 classification, blood pressure, dental hygiene education

Citation: Thompson AL, Collins MA, Downey MC, Herman WW, Konzelman Jr JL, Ward ST, Hughes CT. Prevalence and Severity of Hypertension in a Dental Hygiene Clinic. J Contemp Dent Pract 2007 March;(8)3:013-020.

\section{Introduction}

Determination of blood pressure for dental and dental hygiene patients is an essential step in the assessment phase of care. As a result of various hypertension studies, it has been emphasized hypertension is unquestionably a contributing risk factor in many vascular diseases. Hypertension plays a significant role in the progression of heart failure, kidney failure, stroke, and heart attack. ${ }^{1-3}$

According to the Seventh Report of the Joint National Committee on the Prevention, Detection, Evaluation, and Treatment of High Blood Pressure (JNC7), patients are considered hypertensive if their systolic pressure is equal to or greater than $140 \mathrm{mmHg}$, if their diastolic pressure is greater than or equal to $90 \mathrm{mmHg}$, or if they are presently taking antihypertensive medications. 'About one out of five Americans has hypertension. ${ }^{4}$ It is estimated hypertension precedes the development of congestive heart failure in $91 \%$ of cases. High blood pressure is associated with a two to three times higher risk of developing congestive heart failure. ${ }^{5}$

Researchers have indicated the prevalence of high blood pressure in persons living in the southeast is greater than in any other area of the country, and African Americans develop high blood pressure earlier in life and have a higher average blood pressure than Caucasians. As a result, African Americans have a higher rate of stroke, stroke fatalities, heart disease, and endstage kidney disease. ${ }^{1}$

Previously, it was believed once a patient's blood pressure reading was greater than 140/90 $\mathrm{mmHg}$, the patient was at an increased risk to develop cardiovascular disease. Recent findings published in the JNC7 report present a new "prehypertension" stage. The JNC7

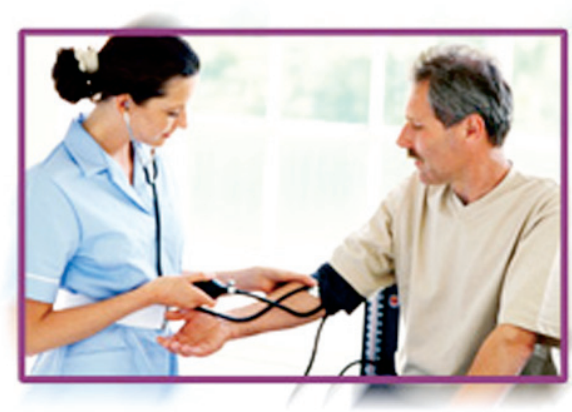

report suggests individuals with a systolic blood pressure of 120 to $139 \mathrm{mmHg}$ or a diastolic blood pressure of 80 to $89 \mathrm{mmHg}$ should be considered at risk for development of hypertension. Lifestyle modifications to promote health should be recommended by healthcare providers. ${ }^{1}$

In 1982 the American Dental Hygienists' Association endorsed the practice of measuring blood pressure on all patients. Standards were developed to include blood pressure as part of the general health assessment data routinely recorded. ${ }^{6}$ In the late nineties dentists were encouraged to take a primary role on the multidisciplinary team involved in treating and educating patients at risk for hypertension. Glick stated professional duties should not be limited to the provision of dental care as oral healthcare providers can have a significant impact on the prevention, detection, evaluation, and treatment of patients with high blood pressure. He further challenged dentists to take a proactive part in the overall healthcare of their patients. Several investigators have encouraged dental hygienists to routinely monitor patients' blood pressure as a part of total patient care. ${ }^{2,6,7}$ These researchers have recognized dental hygienists as specialists in prevention with the education and knowledge necessary to transmit an appreciation of hypertension control as a lifetime duty to all their patients. 
During the medical history update, dental caregivers can identify some of the possible symptoms or signs of hypertension. These include dizziness, fainting, headaches, shortness of breath, memory impairment, and disturbances with concentration. ${ }^{5}$ However, because there are often no symptoms many patients are not aware of increases in their blood pressure. ${ }^{1}$

The first step in the dental hygiene process of care is assessment. This assessment includes gathering pertinent information such as vital signs (blood pressure, pulse, and tobacco use), examining extra oral and intra oral tissue, and performing dental and periodontal chartings. This data is important for the dental hygiene diagnosis. ${ }^{3}$ At this point, the dental hygienist identifies the patients' problems and selects interventions that will achieve the desired outcomes. During the development of the treatment plan, dental hygienists must consider the impact the patients' medical condition(s) may have on the care they will provide.

There are a few dental complications associated with hypertension. Patients with severe hypertension have been reported to develop facial palsy or to bleed excessively after surgical procedures or trauma, but the occurrence of these complications is rare. Side effects of medications used to control hypertension include xerostomia, lichenoid reactions, gingival overgrowth, severe bleeding, and delayed healing. ${ }^{8}$

Uncontrolled hypertension increases a patient's danger of suffering an episode of angina, myocardial infarction, or stroke while in the dental chair. ${ }^{9}$ During dental hygiene appointments, it may be necessary to anesthetize the patient for comfort. The danger in using a local anesthetic containing epinephrine or other vasoconstrictors on a patient with hypertension or other cardiovascular disease is an increase in blood pressure or the development of an arrhythmia. To prevent serious complications, it is unwise to perform elective dental procedures on patients with severe or uncontrolled hypertension. ${ }^{1,8}$ In a recent publication Herman et al. ${ }^{9}$ support this intent in the dental practice setting. They suggest blood pressure readings should be taken on all new patients and annually on recall patients. In addition, they recommend the blood pressure of patients with documented hypertension be assessed at each dental visit because failure to detect severe elevations of blood pressure can result in stroke or myocardial infarction during complex dental procedures.

Since the initiation of the Medical College of Georgia Dental Hygiene program in September 1967 , it has been a common practice for dental hygiene students to record the blood pressure readings of patients previously diagnosed with hypertension. This standard of care became a practice for all adult patients in the mid-seventies. Currently, dental hygiene students learn essential information about hypertension and blood pressure determination during their first semester. They begin applying this knowledge by taking blood pressure readings on each other, and in subsequent semesters they record blood pressure readings on all patients age 16 and older. The probability of detecting an elevated reading in a dental hygiene patient is high. To better identify the hypertensive status of this pool of patients, it is necessary to investigate the prevalence and severity of hypertension in the dental hygiene education environment. It is also essential to study this particular group to determine if further patient education or screening measures should be implemented to provide a complete dental hygiene evaluation. An assessment of current practices is crucial. Dental hygienists have the opportunity to educate their patients regarding hypertension, especially those who are at risk. Therefore, the purpose of this study is to document the prevalence and severity of hypertension in a dental hygiene clinic.

\section{Methods and Materials}

The design of this study was a non-experimental retrospective chart review. Approval was obtained from the Medical College of Georgia Institutional Review Board to conduct the study. Patient records are confidential and were used only for the purposes stated in this protocol. Only records of adult patients treated in the Dental Hygiene Clinic in 2003 were reviewed. This entire accessible population was included to form a sample size of 615 records.

Blood pressure readings used in the study were those previously measured by dental hygiene students during patient care. To measure blood pressure, students used an aneroid 
sphygmomanometer and a stethoscope. The manual procedure for assessing the blood pressure of patients in the dental hygiene clinic is a reliable and validated method. It is outlined in the literature by Cline and Springstead ${ }^{6}$ and widely used by healthcare providers. Utilizing one dental hygiene faculty member to teach the didactic concepts of blood pressure determination and interpretation also enhances the reliability of the blood pressure assessment by dental hygiene students. Clinical competency in assessing blood pressure is evaluated using written didactic testing and clinical skill forms.

\section{Data Collection}

A single researcher extracted from patient records demographic data including race, age, gender, marital status, and occupation based on Takashima et al. ${ }^{10}$ Medical information was also collected. This included the most recent systolic and diastolic blood pressure, diabetes (yes/no), renal disease (yes/no), smoking habits (yes/no), and use of antihypertensive medication (yes/no). Only data from the most recent visit was gathered in this study since many patients had multiple visits in 2003 .

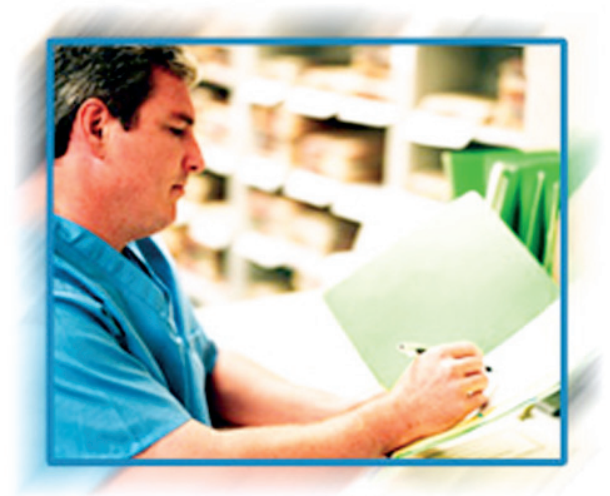

\section{Statistical Analysis}

The level of significance for this study was set at $p<.05$. Cross tabulations of JNC7 classification and categorical modifiers (occupation, marital status, and smoking habits) and non-modifiers (race, gender, and age) were performed using Statistical Package for Social Sciences (SPSS) Version 10.0 software (SPSS Inc., Chicago, IL, USA).

\section{Research Questions}

While the principal research question was "What is the prevalence and severity of hypertension in a dental hygiene clinic?," the goal of this study was to answer the following research questions regarding the dental hygiene clinic policy for the assessment and interpretation of blood pressure readings according to the recent $\mathrm{JNC} 7$ classification of adult hypertension:

1. What is the prevalence and severity of hypertension in patients treated in the dental hygiene clinic at the Medical College of Georgia according to the JNC7 classification?

2. Is there a difference between various nonmodifiers (race, gender, and age) in terms of JNC7 classification?

3. Is there a difference between various modifiers (marital status, smoking habits, and occupation) in terms of JNC7 classification?

4. Is there a difference between subject groups (with/without diabetes and with/without renal disease) in terms of JNC7 classification?

5. Is existing dental hygiene clinical policy for assessing blood pressure and interpreting blood pressure readings consistent with the new JNC7 classification?

\section{Results}

The study sample was approximately $77 \%$ $(615 / 800)$ of the patients treated in 2003. Information from the records of 615 subjects was gathered for this study. The age of the subjects ranged from 18 to 90 years with a median age of 49.5 and mean age of 49.4 years, $S D=18.25$.

The ethnic composition of the sample was African American ( $n=178,29.6 \%)$, Caucasian $(n=380,63.2 \%)$, and a combined group of Asians, Hispanics, multiracial, and others ( $n=43,7.2 \%)$. Fourteen records did not include race, therefore, they were excluded from the analysis of blood pressure by race.

Gender representation was 63.1\% (388/615) female and $36.9 \%(227 / 615)$ male. More than half of the subjects were married $(53.1 \%), 31.8 \%$ were single, $6.5 \%$ were divorced, and $5 \%$ were widowed.

Table 1 indicates the occupation category of subjects. The majority of the subjects were nonsmokers $(86.3 \%, 531 / 615)$. Smokers comprised $13.7 \%(84 / 615)$ of the subjects. Seventy-three $(11.9 \%)$ of the 615 subjects were diabetic and $177(28.8 \%)$ subjects reported use of antihypertensive medication. 
Results indicated the blood pressures of 154 $(25 \%)$ subjects were optimal, while those of 374 (60.8\%) were prehypertensive and 87 (14.1\%) were Stage 1 hypertensive. There were no blood pressure readings with the Stage 2 hypertension classification ( $\geq 160 / \geq 100)$.

A total of 177 (29\%) subjects were taking an antihypertensive. Of those, $40(23 \%)$ were in the normal or optimal blood pressure stage and 111 $(63 \%)$ were in the prehypertensive stage. Even if being treated for hypertension, these two groups were considered at goal or controlled in terms of their readings for that day. The other $26(14 \%)$ subjects taking medication for hypertension had readings in Stage 1 of hypertension; therefore, they were not at goal or controlled for that day.

Results using the chi-square statistic showed the JNC7 classification did not significantly differ between subject groups when considering gender $(p=.23)$ and marital status $(p=.25)$. Results from a one way analysis of variance (ANOVA) showed no significant difference in the JNC7 classification and age $(p=.552)$.

A statistically significant difference in the JNC7 classification was noted between subject groups when considering the non-modifier, race $(p=.02)$, as shown in Table 2. More African Americans had readings at the prehypertension or hypertension Stage I level.

There was also a statistically significant difference in the JNC7 classification of subjects when considering the modifiers smoking habits $(p=.03)$ and occupation $(p=.01)$. Table 3 shows the majority of smokers had prehypertensive or hypertensive Stage I readings. Figure 1 illustrates a greater number of readings in the prehypertension and Stage I hypertension of retired subjects, service personnel, and professional/technology occupations when compared to other occupations.

Table 1. Occupation categories of the subjects.

\begin{tabular}{|l|c|c|}
\hline \multicolumn{1}{|c|}{ Category } & Number & Percent \\
\hline Trans port and communications & 19 & 3.1 \\
\hline Clerical personnel & 41 & 6.7 \\
\hline Managerial and civil personnel & 55 & 8.9 \\
\hline Professional and technical pers onnel & 88 & 14.3 \\
\hline Service personnel & 100 & 16.3 \\
\hline Retired & 136 & 22.1 \\
\hline Unemployed & 44 & 7.2 \\
\hline Student & 60 & 9.8 \\
\hline Not reported & 72 & 11.7 \\
\hline
\end{tabular}

Note: $\mathrm{N}=615$.

Table 2. JNC7 classification and race.

\begin{tabular}{|l|c|c|c|c|}
\hline \multicolumn{1}{|c|}{ Race } & $\begin{array}{c}\text { Normal } \\
<\mathbf{1 2 0 / < 8 0}\end{array}$ & $\begin{array}{c}\text { Prehypertension } \\
\mathbf{1 2 0 - 1 3 9 / 8 0 - 8 9}\end{array}$ & $\begin{array}{c}\text { Stage 1 } \\
\text { Hypertension } \\
\mathbf{1 4 0 - 1 5 9 / 9 0 - 9 9}\end{array}$ & $\begin{array}{c}\text { Total Number of } \\
\text { Subjects in Each } \\
\text { Group }\end{array}$ \\
\hline African-American & $39(21 \%)$ & $115(64.6 \%)$ & $24(13.5 \%)$ & 178 \\
\hline Caucasian & $103(27.1 \%)$ & $228(60 \%)$ & $49(12.9 \%)$ & 380 \\
\hline Other ${ }^{a}$ & $10(23.3 \%)$ & $20(46.5 \%)$ & $13(30.2 \%)$ & 43 \\
\hline $\begin{array}{l}\text { Total } \\
\text { All groups }\end{array}$ & $152(25.3 \%)$ & $363(60.3 \%)$ & $86(14.3 \%)$ & \\
\hline
\end{tabular}

Note: ${ }^{a}$ This group was collapsed for statistical analysis and includes subjects who are Hispanic, Asian, multiracial, and races reported as 'other' in the clinic record. ${ }^{b}$ Result of Chi square analysis reveals the difference between groups is statistically significant: $X^{2}(4, N=601)=11.51, p=0.02$. 
Table 3. JNC7 classification and smoking.

\begin{tabular}{|l|c|c|c|c|}
\hline \multicolumn{1}{|c|}{ Smoking } & $\begin{array}{c}\text { Normal } \\
<120 /<80\end{array}$ & $\begin{array}{c}\text { Prehypertension } \\
\mathbf{1 2 0 - 1 3 9 / 8 0 - 8 9}\end{array}$ & $\begin{array}{c}\text { Stage 1 } \\
\text { Hypertension } \\
\mathbf{1 4 0 - 1 5 9 / 9 0 - 9 9}\end{array}$ & Total \\
\hline No & $142(26.7 \%)$ & $313(58.9 \%)$ & $6(14.3 \%)$ & $531(86.3 \%)$ \\
\hline Yes & $12(14.3 \%)$ & $61(72.6 \%)$ & $11(13.1 \%)$ & $84(13.7 \%)$ \\
\hline
\end{tabular}

Note: ${ }^{a}$ Result of Chi square analysis reveals the difference between groups is statistically significant: $\mathrm{X}^{2}$ $(2, N=615)=6.8, p=0.03$.

Table 4. JNC7 classification and diabetes.

\begin{tabular}{|l|c|c|c|c|}
\hline \multicolumn{1}{|c|}{ Diabetes } & $\begin{array}{c}\text { Normal } \\
<120 /<80\end{array}$ & $\begin{array}{c}\text { Prehypertension } \\
120-139 / 80-89\end{array}$ & $\begin{array}{c}\text { Stage 1 } \\
\text { Hypertension } \\
\mathbf{1 4 0 - 1 5 9 / 9 0 - 9 9}\end{array}$ & Total \\
\hline Yes & $12(16.4 \%)$ & $39(53.4 \%)$ & $22(30.1 \%)$ & $73(12 \%)$ \\
\hline No & $139(26.1 \%)$ & $331(62.1 \%)$ & $63(11.8 \%)$ & $533(88 \%)$ \\
\hline
\end{tabular}

Note: Result of Chi square analysis reveals the difference between groups is statistically significant: $\mathrm{X}^{2}$ $(4, \mathrm{~N}=606)=18.54, \mathrm{p}=0.00$.

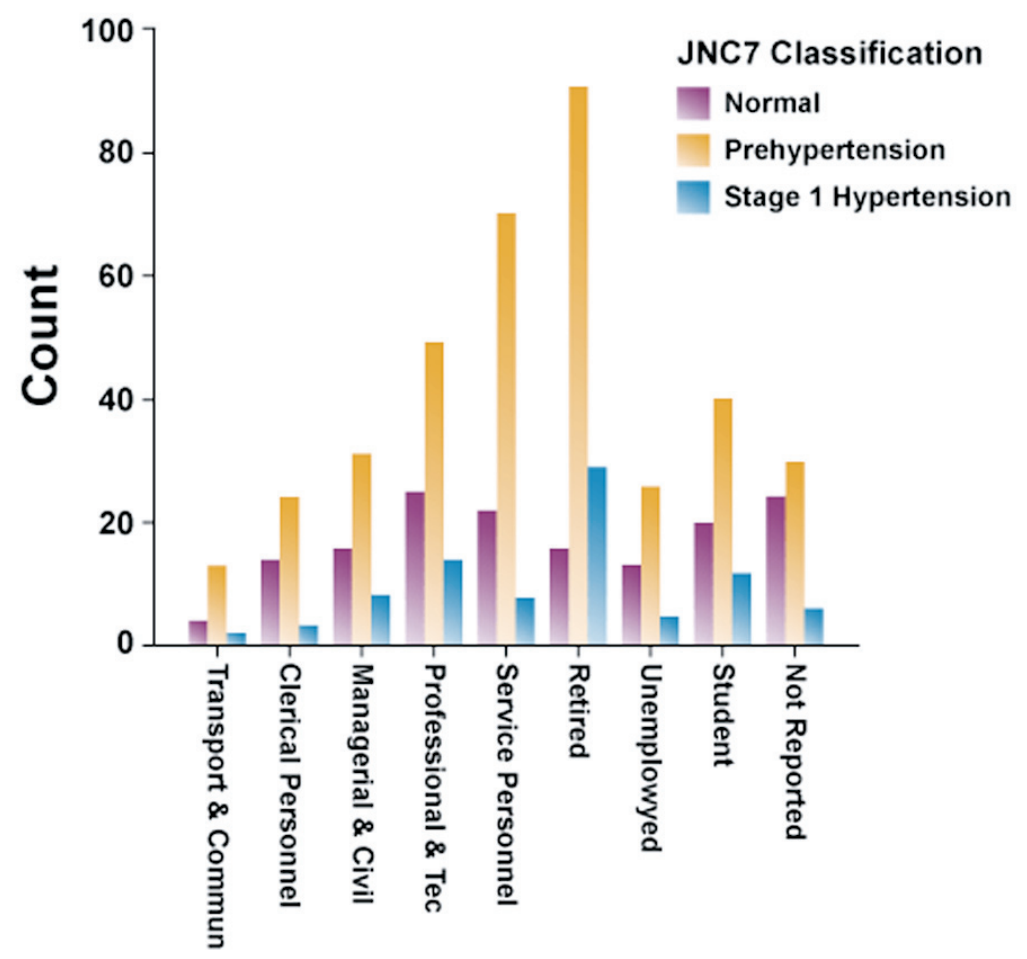

\section{Occupation Category}

Figure 1. JNC7 classification and occupation category.

As shown in Table 4, 22 (30\%) of the diabetic subjects were not at goal or controlled and 39 $(53.4 \%)$ had prehypertensive readings. This difference was statistically significant $(p=.00)$.

\section{Discussion}

Our results closely mirror those published in the literature which show a significant difference in hypertension prevalence when considering race, smoking habits, occupation, and diabetes. .,2,5,7-10 $^{\circ}$

Consistent with the current study, statistics from the American Heart Association show African Americans have a higher average blood pressure than Caucasians. ${ }^{4}$ Several authors also agree current smokers are at risk for systolic 
hypertension. ${ }^{1,9}$ Unlike Zachariah et al., ${ }^{11}$ the results of the present study found occupations involving moderate or greater physical activity was inversely associated with the prevalence of hypertension. In their study, more sedentary employees like office workers had more hypertension when compared to more physically active factory and assembly line workers.

A particularly alarming finding in the current study shows $30 \%$ of diabetic subjects were not at goal or controlled. Kaplan noted the combination of diabetes and hypertension adds to cardiovascular and renal damage, placing all diabetic hypertensives at increased risk for these conditions. ${ }^{12}$

The most recent JNC7 guidelines are encouraging patients to maintain blood pressure readings lower than what it was considered normal a few years ago. The majority of records reviewed in this study reflected readings no longer considered normal. Even though the results of this study cannot be generalized to the entire population, they can be useful when planning modifications to the current dental hygiene clinic policy on assessing and interpreting blood pressure readings. The findings of this research project reiterate what has been proposed by the JNC7 and other authors who place emphasis on the need of increasing patients' awareness about the dangers of hypertension.

The diagnosis of hypertension must be made by a physician, usually after several abnormal readings are documented. However, dental hygiene students are in a unique position to assess blood pressure and increase the patient's awareness of the potential risks associated with hypertension including obesity, smoking, and a sedentary lifestyle.
Most encounters with allied health professionals occur when patients are ill or undergoing diagnostic procedures, but since a systemic illness is not the reason for seeking dental hygiene care many patients consider themselves healthy during their dental visits. It is common for dental hygienists to treat medically compromised patients. In contrast to other allied healthcare professionals dental hygienists have regular contact with patients due to frequent care intervals. For that reason, dental hygienists have an exceptional opportunity to monitor blood pressure. Communication concerning high readings and appropriate patient education may motivate patients to seek the care of their physicians.

\section{Conclusion}

Based on the results of this study, the researchers suggest clinical policy modifications including: additional documentation for blood pressure readings in the prehypertension stage, lowering the systolic readings from $160 \mathrm{mmHg}$ to 140 $\mathrm{mmHg}$ when adding hypertension alert labels, and documenting prehypertension/hypertension on the dental hygiene care plan with the appropriate intervention such as medical consultation prior to dental and dental hygiene treatment.

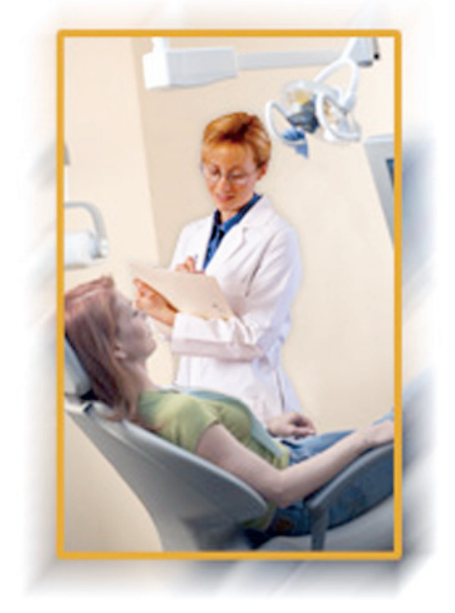




\section{References}

1. Chobanian AV, Bakris GL, Black HR, Cushman WC, Green LA, Izzo Jr. JL, Jones DW, Materson BJ, Oparil S, Wright Jr. JT, Rocella EJ, and the National High Blood Pressure Education Program Coordinating Committee. JNC7-Complete Version. Seventh Report of the Joint National Committee on Prevention, Detection, Evaluation, and Treatment of High Blood Pressure. Hypertension 2003;42:1206-52.

2. Hughes $\mathrm{CT}$, Thompson AL, Browning WD. Blood pressure screening practices of a group of dental hygienists. J Dent Hygiene 2004;78(4).

3. Wyche CJ. The dental hygiene care plan. In E.M. Wilkins Clinical practice of the dental hygienist. Ninth ed. Philadelphia: Lippincott Williams \& Wilkins, 2005:351-61.

4. American Heart Association. Heart disease and stroke statistics: 2003 update. Dallas.

5. Levy D, Larson MG, Vasan RS, Kannel WB, Ho KK. The progression from hypertension to congestive heart failure. [comment] J Am Med Assoc 1996;275(20):1557-62.

6. Cline NV, Springstead MC. Monitoring blood pressure. Five minutes critical to quality patient care. J Dent Hygiene 1992;66(8):363-8.

7. Glick M. New guidelines for prevention, detection, evaluation and treatment of high blood pressure. J Am Dent Assoc 1998;129(11):1588-94.

8. Little JW, Falace DA, Miller CS, Rhodus NL. Dental management of the medically compromised patient. 6th Edition, St. Louis: Mosby, 2002.

9. Herman WW, Konzelman JL, Prisant LM. New national guidelines on hypertension. A summary for dentistry. J Am Dent Assoc 2004;135:576-84.

10. Takashima Y, Yoshida M, Kokase A, Orido Y, Tsugane S, Ishikawa M, Takeuchi Y, Takagi Y, Tanaka N, Watanabe S, Akamatsu T. Relationship of occupation to blood pressure among middle-aged Japanese men- the significance of the differences in body mass index and alcohol consumption. $J$ Epidemiol, 1998; 8(4), 216-226.

11. Zachariah MG, Thankappan KR, Alex SC, Sarma PS, Vasan, RS. Prevalence correlates, awareness, treatment, and control of hypertension in a middle-aged urban population in Kerala. Indian Heart $\mathrm{J}$, 2003; 55(3) 245-251.

12. Kaplan NM. Hypertension and diabetes. J Hum Hypertens, 2002;16, Suppl. 1, 56-60. 
About the Authors
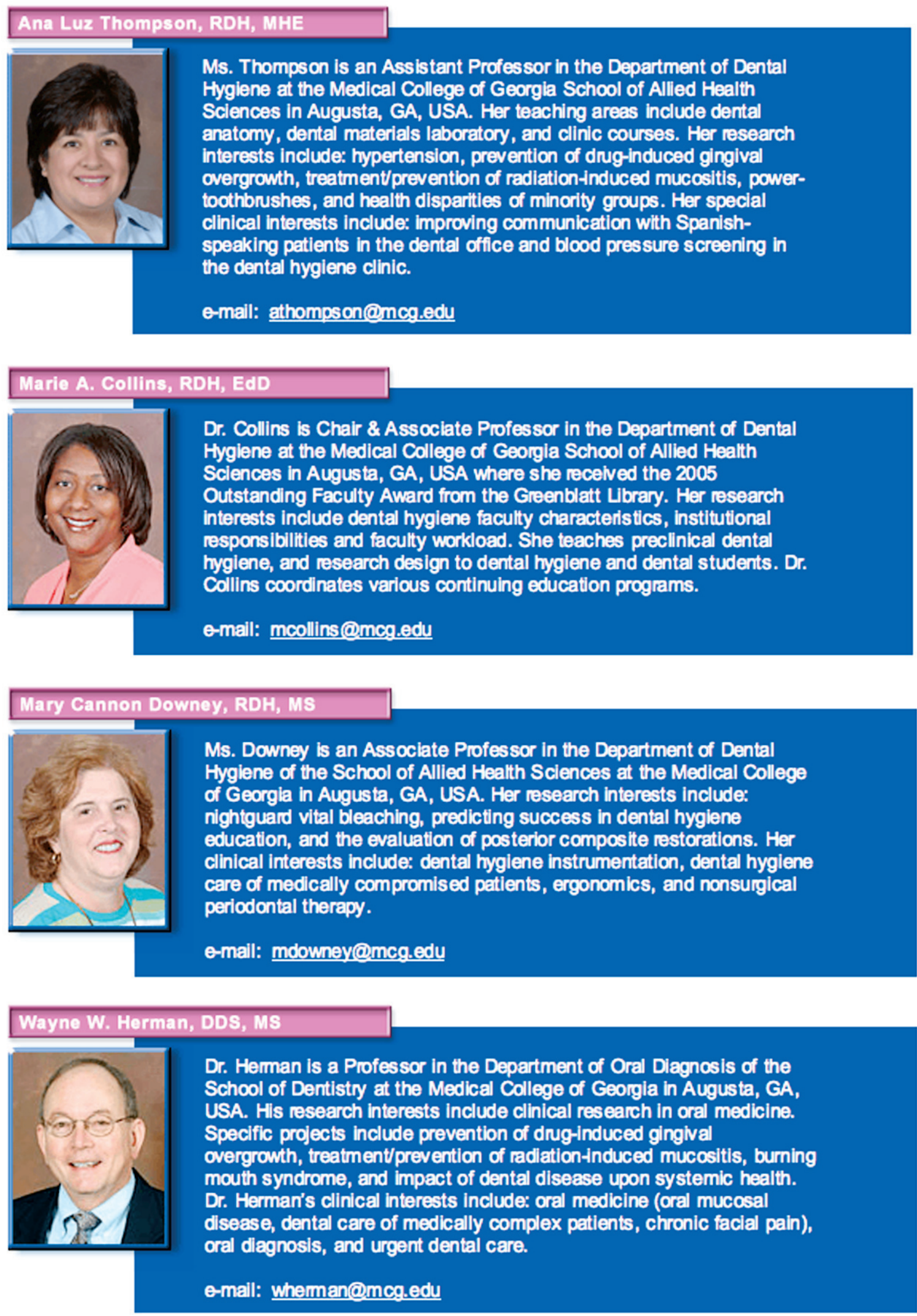

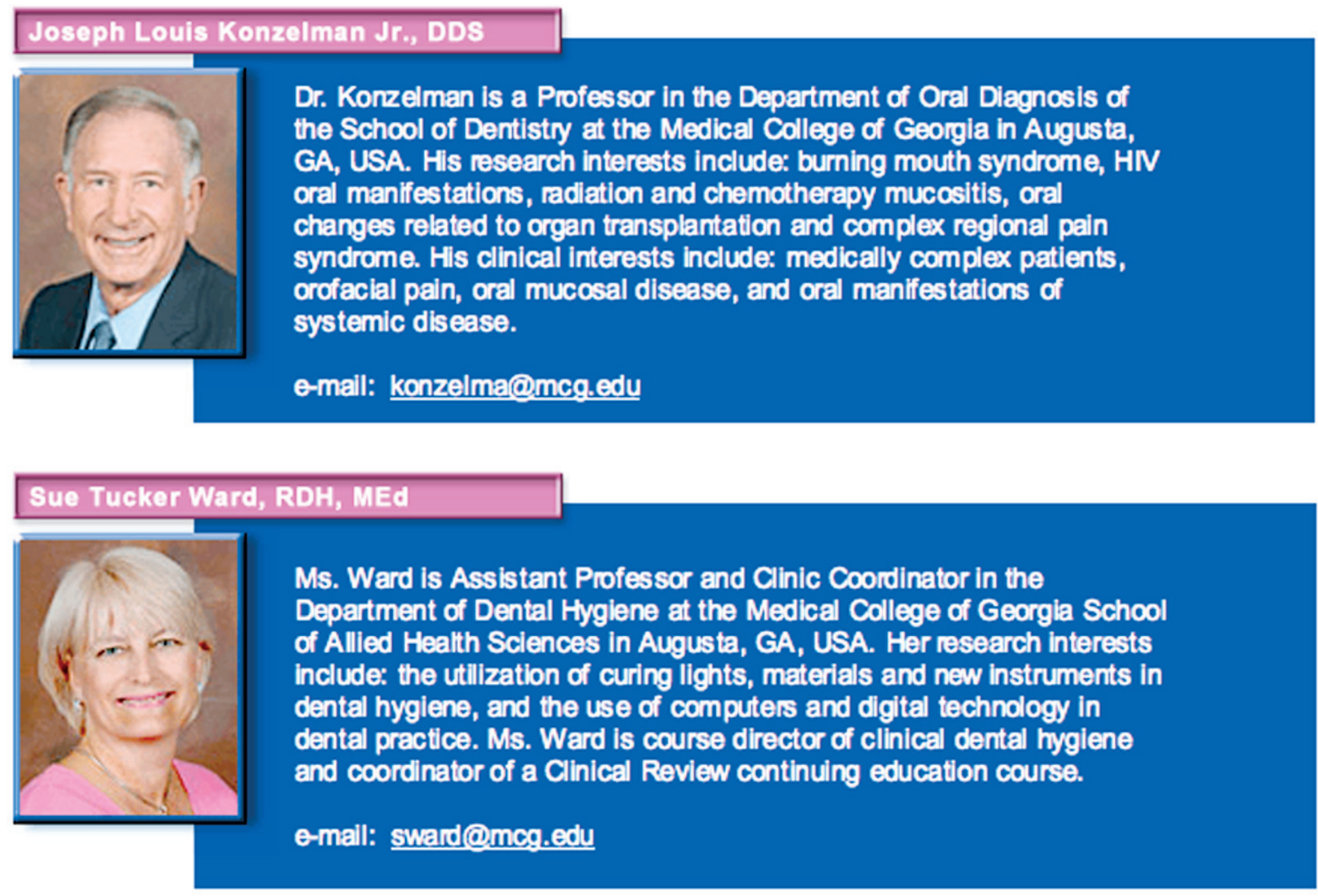

\section{Cynthia T. Hughes, RDH, BSDH, MEd}

Ms. Hughes recelved her dental hyglene degree from the Medical
College of Georgla and her Master of Education from Cambridge
College. She has over twenty years experience as a cliniclan, educator,
and speaker. Her research interests include the performance of curing
lights and blood pressure screening. She has served as an instructor for
the Medical College of Ceorgla Department of Dental Hyglene in
Augusta, GA, USA and is currently a corporate dental hygiene
administrator for CoastDental in Tampa, FL, USA.
e-mail: chughes@coastdental.com

